\title{
Trajectories in the complex phase space and quantization by numerical contour integration
}

\author{
A. Nanayakkara ${ }^{*}$ \\ Department of Physics, University of Sri Jayawardanapura, Gangodawila, Nugegoda, Sri Lanka.
}

\begin{abstract}
In this paper an exact numerical quantization method, based on quantum action variable theory is presented. The quantum momentum function (QMF) is redefined such that the corresponding quantum action variable (QAV) is an integer $(\hbar=1)$ for energy eigenvalues and non integer for off eigenvalues. The energy eigenvalues obtained by quantizing the quantum action variable are exact up to the accuracy of the numerical methods used. One of the major advantages of this method is that one could use interpolations or extrapolations for obtaining boundstate energies from a set of QAV values corresponding to non-eigen energies. Examples are given to illustrate the new numerical method and the interpolation and extrapolation methods.
\end{abstract}

\section{INTRODUCTION}

In WKB theory the semiclassical eigenvalues are obtained by the condition

$$
J_{c} \equiv \int p_{C} d x=\left(n+\frac{1}{2}\right) \hbar
$$

where $n=1,2, \ldots, J_{c}$ is the classical action variable ${ }^{1}$, and $p_{c}$ is the classical momentum function given by $p_{c}=(E-v(x))^{1 / 2}$. The integration is carried over a complete period. The quantum action variable ${ }^{2,3}$, the quantum analogue of the classical action variable, is defined as a contour integral

\footnotetext{
${ }^{*}$ Corresponding author
} 


$$
J=\frac{1}{2 \pi} \int_{C} p(x) d x
$$

where the quantum momentum function (QMF), $p(x)$, is the solution of the quantum momentum function equation (QMF equation),

$$
\frac{h}{i}\left(\frac{d p(x)}{d x}\right)+p^{2}(x)=p_{c}^{2}(x)
$$

The contour $\mathrm{C}$ in equation (2) encloses the two physical turning points of $p_{c}(x)$. The bound state boundary condition imposed upon QMF $p(x)$ is

$$
p(x) \rightarrow p_{c}(x) \quad \text { as } h \rightarrow 0 .
$$

Since the quantum momentum function $p(x)$ is related to the wavefunction $\psi(x)$ by

$$
p(x)=\frac{\hbar}{i} \frac{\frac{\partial \psi(x)}{\partial x}}{\psi(x)}
$$

the contour integral (2) counts the number of zeros of $\psi(x)$ (i.e. number of poles of $p(x)$ ). As there are finite number of zeros of $\psi(x)$, and hence finite number of isolated poles of $p(x)$, lie between two physical turning points for both the eigen and off eigenvalues of $E$ 3 , unlike in the semiclassical (WKB) case, the quantum action variable $J$ is an integer (assuming $\hbar=1$ ) for both the eigen and off eigenvalues of $E$. Therefore the definition of the quantum action variable in references ${ }^{2,3}$ cannot be directly employed for numerical calculations on boundstate energy eigenvalues.

In this paper the quantum momentum function is redefined such that the corresponding quantum action variable is an integer $(\hbar=1)$ for eigenvalues and noninteger for off eigenvalues. This property is then applied for quantizing the quantum action variable numerically. Since the QAV $J$ varies smoothly between eigenvalues, by calculating $J$ at different energies (not necessarily be at eigen values) approximate bound state eigenvalues can be calculated by interpolation or extrapolation. These energies can 
be employed as starting values for more accurate calculations when higher accuracy is desired.

\section{THEORY / NUMERICAL METHOD}

In order to develop a numerical method based on the quantum action variable theory to calculate bound state energies, first we redefine the quantum momentum function (denoted by $P(x)$ ) by rewriting the equation (1) in the form with the boundary condition $P(x) \rightarrow P_{c}(x)$ as $\hbar \rightarrow 0$ and then we solve the equation (6)

$$
\mathrm{P}(x)=\sqrt{P_{c}^{2}(x)+i \hbar \frac{\partial \mathrm{P}(x)}{\partial x}}
$$

iteratively, starting with $P(x)=P_{c}(x)$ and $\frac{\partial P(x)}{\partial x}=\frac{\partial P_{c}(x)}{\partial x}$ along the contour C. After each iteration $\frac{\partial P(x)}{\partial x}$ is calculated numerically and then substitute it back in the equation (6) to obtain new $P(x)$. The convergence of $P(x)$ for a given energy is tested by calculating the quantum action variable $J_{P}$ (by equation (7)) and checking its convergence.

$$
J_{\mathrm{P}}=\frac{1}{2 \pi} \int_{C} \mathrm{P}(x) d x
$$

In this study we found that unlike the quantum action variable defined by the equation (2), the new quantum action variable $J_{P}$ is an integer for eigenvalues and noninteger for off-eigen values. The reason for this difference is that the branch introduced by the square root in equation (6) produces non zero contribution to the contour integral (7) for off-eigenvalues while it produces zero contribution for the eigenvalues. Note that unlike the previous QMF, the new QMF is a multivalued function of $\mathrm{x}$ and does not satisfy the equation (5). There are many ways to construct closed contours in the complex x-plane to enclose the physical turning points of classical momentum function $P_{c}(x)$. However, one has to be careful not to include any non-physical turning points or 
poles of $P_{c}(x)$ inside the contour C.

In this study we found that the rapid convergence of $J_{P}$ can be achieved by using 2-D projection of a classical trajectory on the complex coordinate plane (i.e. Complex $\mathrm{x}$ plane.) as the contour. If the starting point of the trajectory is close enough to one of the physical turning points of $P_{c}(x)$ and it is complex then the trajectory will enclose the other turning point as well (see figure (1)). This way we obtain closed contours which enclose two physical turning points of $P_{c}(x)$ and the values of $P_{c}(x)$ at the discreet points on the contours. $\frac{\partial P}{\partial x}$ is calculated at each discreet point on the contour, from $P(x)$ by the following equations,

$$
\begin{gathered}
\frac{\mathrm{P}\left(x_{i+1}\right)-\mathrm{P}\left(x_{n}\right)}{2 \Delta x}, \text { if } i=1 \\
\frac{\partial \mathrm{P}\left(x_{i}\right)}{\partial x_{i}}=\frac{\mathrm{P}\left(x_{1}\right)-\mathrm{P}\left(x_{i-1}\right)}{2 \Delta x}, \text { if } i=n \\
\frac{\mathrm{P}\left(x_{i+1}\right)-\mathrm{P}\left(x_{i-1}\right)}{2 \Delta x}, \text { otherwise }
\end{gathered}
$$

where $\mathrm{n}$ is the total number of points on the contour.

In order to calculate $P(x)$ correctly from equation (6) the square root function is redefined such that the $P(x)$ is smooth along the contour.

For the evaluation of the contour integral $J_{P}$,the extended Simpson rule is used ${ }^{4}$. The value of $\left|J_{P}-\left(J_{P}\right)_{\text {old }}\right|$ is compared with a tolerance value to test the convergence of the quantum action variable $J_{P}$. The converged value of $J_{P}$ is then compared with a given integer $n$ to find out whether the energy used in the calculation is the boundstate eigenvalue corresponding to integer $n$. The rational functional approximation is used for interpolation and extrapolation of energies ${ }^{4}$. 


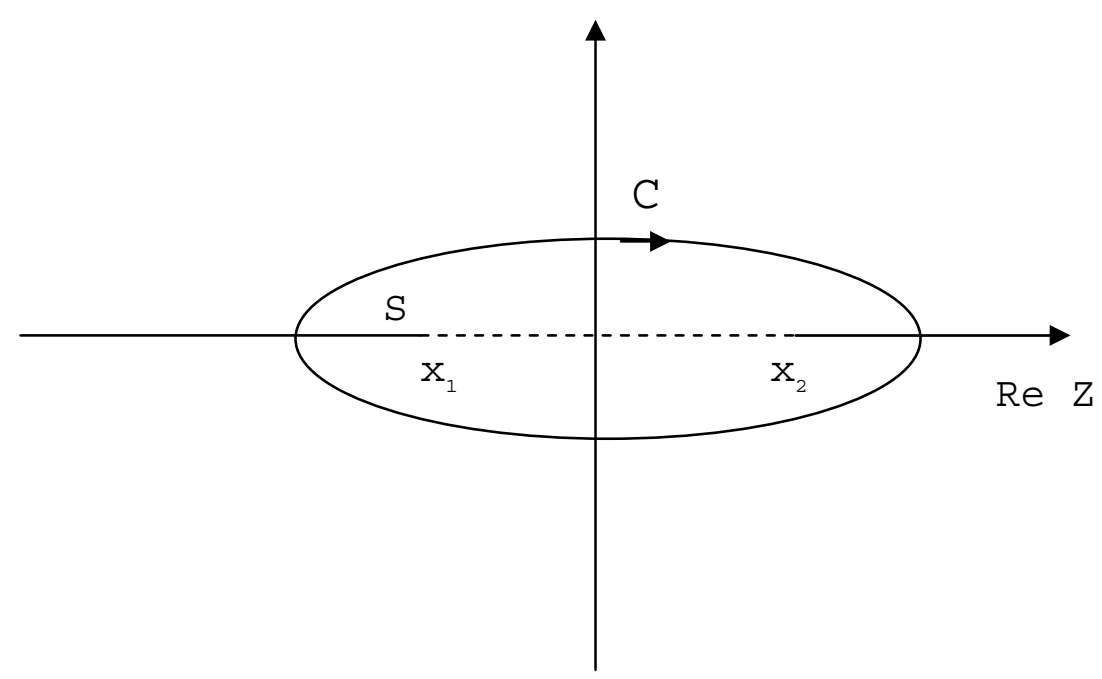

Fig. 1. A typical contour in the complex x plane. $x_{1}$ and $x_{2}$ are the physical turning points. A branch cut of $p_{c}$ (broken line) runs between $x_{1}$ and $x_{2} . S$ is the initial point of the classical trajectory which encloses both turning points.

\section{NUMERICAL ILLUSTRATIONS}

Two examples are given here to illustrate the numerical method and the accuracy of the interpolation and extrapolation techniques described in the last section. The first illustration is the "barrier Oscillator" potential $v(x)=x^{2}+a^{2} / x^{2}$. Since this potential admits analytic solutions ${ }^{1,5}$, the accuracy of our contour integral method can be evaluated. In addition to four turning points, this system has one pole of $P_{c}(x)$ at the origin. In order to exclude the pole, the starting point of the classical trajectory is taken close to the turning point $x_{1}$ (see figure (2)). Then only the two positive turning points $x_{1}$ and $x_{2}$ of $P_{c}(x)$ were enclosed by the trajectory as shown in figure (2).

The exact boundstate energy of this system is given by equation (9).

$$
E=2(2 n+1) \hbar+2 \sqrt{a^{2}+\frac{\hbar^{2}}{4}}
$$


The results of the numerical calculations are presented in table (1). As shown by the data presented in the table, the calculated values of the first six bound state energies are in excellent agreement with the exact values, although the accuracy slightly decreases for higher excited states. Note that for this calculation the Plank constant $\hbar$ is assumed to be 0.001 units. ( small values of $\hbar$ guarantee the convergence of $P(x)$ in the equation (6)).

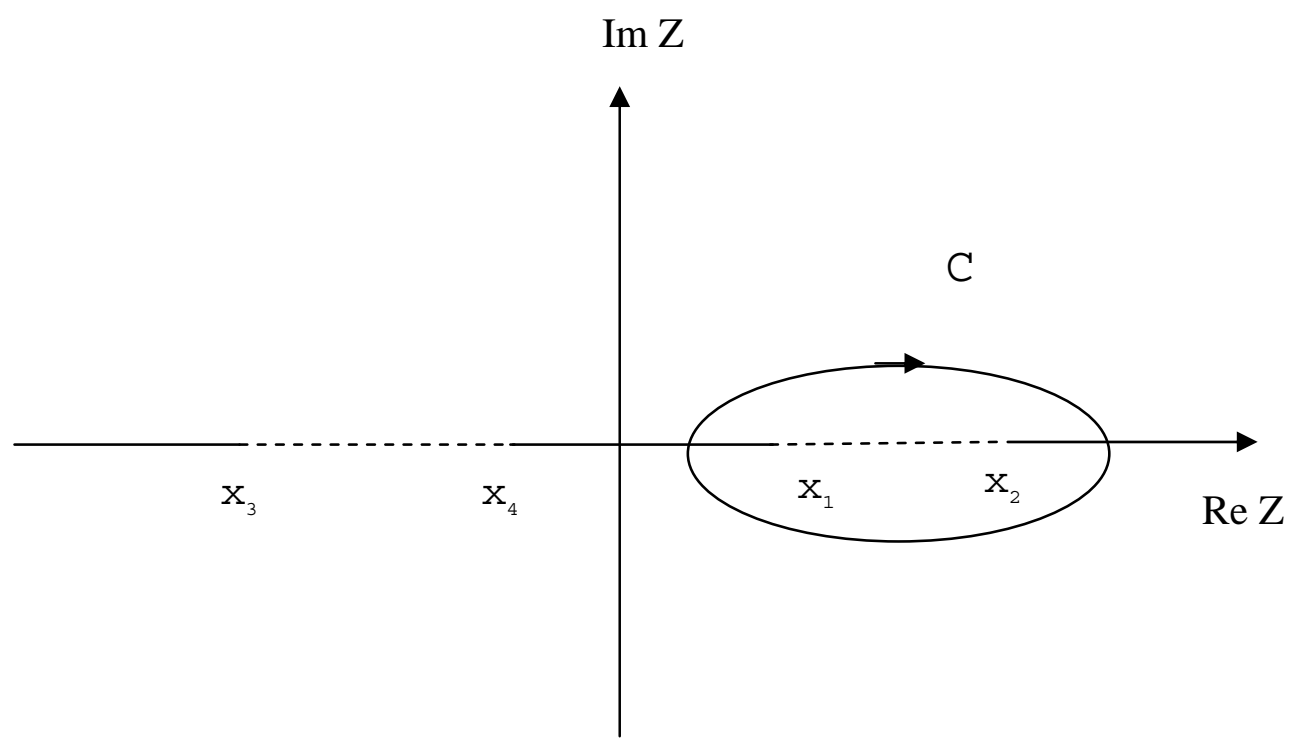

Fig. 2. A contour in the complex $x$ plane for the potential $v(x)=x^{2}+a / x^{2} . x_{1}, x_{2}, x_{3}$, and $x_{4}$ are the four turning points of $p_{c}$. When the initial point $S$ is close to $x_{1}$, the trajectory encloses only the turning points on the right $\left(x_{1}\right.$ and $\left.x_{2}\right)$. The branch cuts of $p_{c}$ are marked with broken lines.

Table 1. $\mathrm{V}(\mathrm{x})=\mathrm{x}^{2}+\mathrm{a}^{2} / \mathrm{x}^{2}$ with $\mathrm{a}=0.1$ and $\hbar=0.001$

\begin{tabular}{|l|l|l|}
\hline $\mathrm{n}$ & Calculated Energy & Exact Energy \\
\hline 0 & 0.6344563226 & 0.6344563226 \\
\hline 1 & 0.6384563227 & 0.6344563226 \\
\hline 2 & 0.6424563228 & 0.6344563226 \\
\hline 3 & 0.6464563229 & 0.6344563226 \\
\hline 4 & 0.6504563228 & 0.6344563226 \\
\hline 5 & 0.6544563228 & 0.6344563226 \\
\hline
\end{tabular}


The second illustration, the anharmonic oscillator potential $v(x)=x^{2}+\lambda x^{4}$ with $\lambda$ $=0.1$ is used to illustrate the capability of the interpolation and extrapolation techniques. This system cannot be solved analytically. This system also has four turning points; two of them are real and physical while the other two are complex. We choose 2-D projection of a complex classical trajectory (on classical co-ordinate space) which include only the two physical turning points of $P_{c}(x)$ as the contour (as shown in figure (3)).

In order to illustrate the accuracy of the interpolation methods for obtaining approximate bound state energies, first we calculate QAV $J$ for 10 different equally spaced eigen energies between 1.0 and 14.0. (Note that none of these values are eigenvalues.)

The corresponding calculated $J$ values are between -0.3066 and 5.01026. Then the first six (i.e. for $n=0,1 . .5$ ) approximate bound state energies are found by interpolation while the last five bound states are found by extrapolation.

The resulting interpolated (or extrapolated) bound state energies together with the accurate bound state energies (calculated as in the first example) are shown in table (2). As shown in table (2), the agreement between accurate energies and the interpolated and extrapolated energies for the first eight eigenvalues are very good. Although the last three extrapolated energy values are not so accurate, they can be taken as starting values for more accurate calculations. The table (3) shows the first eleven interpolated energies using 10 non-eigen values between 1.0 and 30.0. One can see the agreement between new interpolated energies and the accurate energies. As one would expect, interpolation give rise to better values than the extrapolated values.

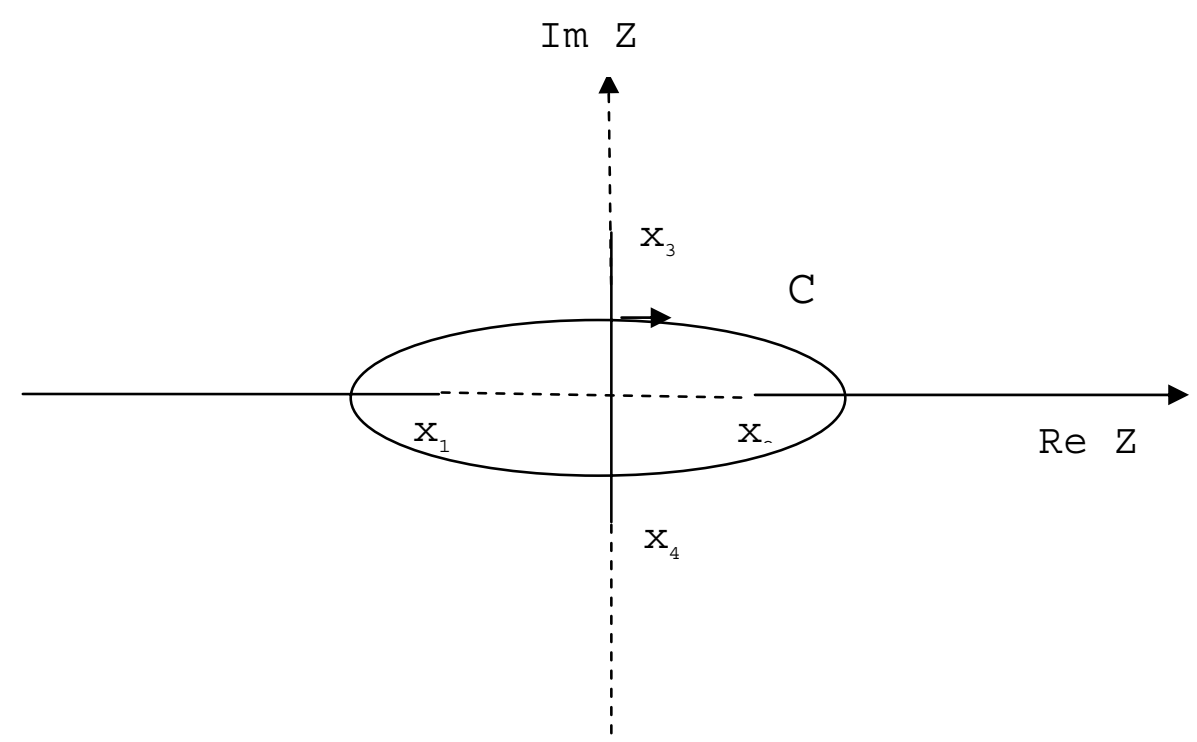

Fig. 3. The points $x_{1}, x_{2}, x_{3}$, and $x_{4}$ are the turning points of $p_{c}$ in the complex $x$-plane for the potential $v(x)$ $=x^{2}+\lambda x^{4}$. The classical trajectory encloses only the real turning points. The branch cuts of $p_{c}$ run between $x 1$ and $x 2, x 3$ and $\infty$, and $x 4$ and $-\infty$. 
Table 2. Comparison between exact energy eigen values and the energy eigen values found by extrapolation / interpolation for the potential $\mathrm{v}(\mathrm{x})=\mathrm{x}^{2}+\lambda \mathrm{x}^{4}$ with $\lambda=0.1$. The data set used for interpolations and extrapolations consists of ten equally spaced energy values between 1.0 and 14.0 along with ten corresponding $J$ values.

\begin{tabular}{|l|l|l|}
\hline $\mathrm{n}$ & $\begin{array}{l}\text { Enegry } \\
\text { (Accurate) }\end{array}$ & $\begin{array}{l}\text { Energy } \\
\text { Interpolated/Extrapolated }\end{array}$ \\
\hline 0 & 1.065285 & 1.065036 \\
\hline 1 & 3.306872 & 3.306863 \\
\hline 2 & 5.747959 & 5.747950 \\
\hline 3 & 8.352677 & 8.352672 \\
\hline 4 & 11.09859 & 11.09859 \\
\hline 5 & 13.96993 & 13.96992 \\
\hline 6 & 16.95474 & $16.95474 *$ \\
\hline 7 & 20.04380 & $20.04360 *$ \\
\hline 8 & 23.22949 & $23.22861 *$ \\
\hline 9 & 26.50549 & $26.50297 *$ \\
\hline 10 & 29.86646 & $29.86059 *$ \\
\hline
\end{tabular}

* These values are calculated by extrapolations.

Table 3. Comparison between exact energy eigen values and the energy eigen values found by interpolation for the potential $v(x)=x^{2}+\lambda x^{4}$ with $\lambda=0.1$. The data set used for interpolations consists of ten equally spaced energy values between 1.0 and 30.0 along with ten corresponding $J$ values.

\begin{tabular}{|l|l|l|}
\hline$n$ & $\begin{array}{l}\text { Energy } \\
\text { (Accurate) }\end{array}$ & $\begin{array}{l}\text { Energy } \\
\text { (Interpolation) }\end{array}$ \\
\hline 0 & 1.06528 & 1.06501 \\
\hline 1 & 3.30687 & 3.30687 \\
\hline 2 & 5.74795 & 5.74795 \\
\hline 3 & 8.35267 & 8.35267 \\
\hline 4 & 11.09859 & 11.09859 \\
\hline 5 & 13.96993 & 13.96992 \\
\hline 6 & 16.95474 & 16.95478 \\
\hline 7 & 20.04380 & 20.04385 \\
\hline 8 & 23.22949 & 23.22952 \\
\hline 9 & 26.50549 & 26.50554 \\
\hline 10 & 29.86646 & 29.86651 \\
\hline
\end{tabular}




\section{DISCUSSION}

The definitions presented in this paper for the quantum momentum function and the quantum action variable enable us to introduce a new numerical method for obtaining bound state energy eigenvalues. Our calculations have clearly demonstrated that this contour integral method can yield accurate bound state eigenvalues. The accuracy of the method mainly depends upon the number of points on the contour and the accuracy of the numerical method used for contour integration. The convergence of the quantum momentum function $P(x)$ depends on the value of $\hbar$ and the accuracy of the numerical method used to calculate $\frac{\partial P(x)}{\partial x}$.

Since the new quantum action variable varies smoothly between eigenvalues, the approximate bound state energies can be obtained by interpolation techniques. We showed in the last section that as few as ten $J$ values corresponding to non-eigen energies can be utilized to obtain eleven (or more) bound state energies with a good accuracy by interpolation. Note that the direct numerical integration of Schrodinger's equation or matrix diagonalization methods cannot be employed as such. This one dimensional contour integral method can be extended for multi dimensional systems using multidimensional quantum action variable theory ${ }^{6}$.

\section{REFERENCES}

1. N. Fröman and P. O.Fröman, JWKB Approximation, (North-Holland, Amsterdam, 1965).

2. R. A. Leacock and M. J. Padgett, Quantum Action Variable theory, Phys. Rev. Lett. 50, 3 (1983).

3. R. A. Leacock and M. J. Padgett, Phys. Quantum Action Variable theory: A new method of solving 1$\mathrm{D}$

Eigen-Value Problems, Phys Rev. D 28, 2491 (1983).

4. W. H. Press, B. P. Flannery, S. A. Teukolsky, and W. T. Vetterling, Numerical Recipes in C (Cambridge

Univesity Press 1988).

5. A. Nanayakkara, Transformations in Quantum Action Variable Theory, J. Phys A: Math. Gen. 23, 2055 (1990).

6. A. Nanayakkara (In progress) 